Check for updates

Cite this: Nanoscale Adv., 2021, 3, 5277

\section{The fragmentation mechanism of gold nanoparticles in water under femtosecond laser irradiation $\dagger$}

\author{
Gabriele Bongiovanni, \$ Pavel K. Olshin, $\$$ Chengcheng Yan, Jonathan M. Voss, \\ Marcel Drabbels and Ulrich J. Lorenz (iD*
}

Received 31st May 2021

Accepted 31st July 2021

DOI: $10.1039 / d 1 n a 00406 a$

rsc.li/nanoscale-advances
Plasmonic nanoparticles in aqueous solution have long been known to fragment under irradiation with intense ultrafast laser pulses, creating progeny particles with diameters of a few nanometers. However, the mechanism of this process is still intensely debated, despite numerous experimental and theoretical studies. Here, we use in situ electron microscopy to directly observe the femtosecond laser-induced fragmentation of gold nanoparticles in water, revealing that the process occurs through ejection of individual progeny particles. Our observations suggest that the fragmentation mechanism involves Coulomb fission, which occurs as the femtosecond laser pulses ionize and melt the gold nanoparticle, causing it to eject a highly charged progeny droplet. Subsequent Coulomb fission events, accompanied by solution-mediated etching and growth processes, create complex fragmentation patterns that rapidly fluctuate under prolonged irradiation. Our study highlights the complexity of the interaction of plasmonic nanoparticles with ultrafast laser pulses and underlines the need for in situ observations to unravel the mechanisms of related phenomena.

Laser irradiation of plasmonic nanoparticles in aqueous solution gives rise to a dizzying array of phenomena that are often difficult to disentangle..$^{1-6}$ The optical forces exerted on the particle find use in optical tweezers ${ }^{7,8}$ and have been employed to stretch particles into elongated shapes. ${ }^{9}$ The excitation of plasmons can drive photochemical reactions of adsorbates on the particle surface. ${ }^{10,11}$ It can also be used to etch or grow the nanoparticles, ${ }^{12}$ processes that are being harnessed in the plasmon-mediated synthesis of triangular gold and silver nanoprisms. ${ }^{13,14}$ Intense laser pulses induce partial or complete melting and have been employed to reshape nanoparticles ${ }^{15}$ or weld them together ${ }^{16,17}$ and thus tailor their plasmonic properties. ${ }^{18,19}$ As the particles dissipate heat, nanobubbles form and

Laboratory of Molecular Nanodynamics, École Polytechnique Fédérale de Lausanne, 1015 Lausanne, Switzerland.E-mail: ulrich.lorenz@epfl.ch

$\dagger$ Electronic supplementary information (ESI) available: Methods, ESI Fig. 1-3, ESI Movies 1-14. See DOI: 10.1039/d1na00406a

\$ These authors contributed equally. explosively expand, eventually collapsing as the particles cool. $^{20-22}$ At even higher fluence, the nanoparticles undergo complex fragmentation processes that involve a range of mechanisms and that vary with the laser pulse parameters. ${ }^{18-20}$

The fragmentation of metallic nanoparticles in water under ultrafast laser irradiation has attracted particular interest since it generates fragments of $2-4 \mathrm{~nm}$ diameter, ${ }^{1,19,20,23}$ providing a simple route to this particular size range..$^{20}$ Fragments have been shown to form within $100 \mathrm{ps}$ after the laser pulse ${ }^{1}$ and to slightly increase in size for several microseconds after their appearance. ${ }^{20}$ In the absence of direct observations, the mechanism of this process has remained contentious though. ${ }^{6,20,21} \mathrm{It}$ has been suggested that it involves a thermal explosion and the subsequent condensation of the metal vapor into smaller particles, ${ }^{20,24}$ in close analogy to the fragmentation mechanism under nanosecond laser irradiation. ${ }^{25-27}$ Since fragmentation occurs even if the nanoparticle temperature barely exceeds the melting point, it was instead proposed that the femtosecond pulses ionize the particle, causing it to Coulomb explode into a large number of charged fragments. ${ }^{1}$ Other mechanisms typically occurring at higher laser intensities have also been considered, ${ }^{20}$ such as near-field ablation ${ }^{28}$ or the shockwavemediated disintegration during impulsive melting. ${ }^{29}$

We recently demonstrated that the femtosecond laserinduced fragmentation of gold nanoparticles encapsulated in a silica shell follows a distinctly different mechanism, ${ }^{30}$ raising the question whether the same may be true in water. Using in situ electron microscopy, ${ }^{31}$ we observed that the gold particles eject individual clusters of about 10-500 atoms $(0.7-2.5 \mathrm{~nm}$ diameter), rather than undergoing an explosion or shattering violently. Instead, our observations provide evidence that the underlying mechanism involves Coulomb fission, ${ }^{32-34}$ which occurs after the ultrafast laser pulse ionizes the gold core in a multiphoton process ${ }^{35,36}$ and melts it. If the Coulomb repulsion in the resulting liquid gold droplet surpasses its surface tension, i.e. the Rayleigh stability limit is exceeded, ${ }^{37}$ a highly charged progeny droplet is emitted along the laser polarization direction. ${ }^{33}$ As the process repeats under prolonged irradiation, 
the ejected clusters accumulate and coalesce into a second core, so that a binuclear particle is formed.

\section{Results and discussion}

Here, we use in situ liquid cell transmission electron microscopy to directly observe the fragmentation of gold nanoparticles in water under femtosecond laser irradiation and elucidate its mechanism. A schematic of the experiment is displayed in Fig. 1a (see Methods $\dagger$ ). A suspension of gold nanoparticles in distilled water (15 and $50 \mathrm{~nm}$ diameter, polyvinylpyrrolidone stabilized) is enclosed in a microchip-based liquid cell with silicon nitride viewing windows. ${ }^{38}$ Particles covered in a thin layer of water (an estimated few hundred nanometers thick, see Methods $\dagger$ ) are irradiated in situ with femtosecond laser pulses ( $515 \mathrm{~nm}, 200 \mathrm{fs}, 10 \mathrm{kHz}$ ), and their fragmentation is observed in real time with the electron beam. ${ }^{46}$ Notably, this allows us to study the process in liquid phase, which is the medium employed in synthetic applications.

Fig. 1b shows a typical micrograph of gold nanoparticles in water. Irradiation with femtosecond laser pulses $\left(53 \mathrm{~mJ} \mathrm{~cm} \mathrm{~cm}^{-2}\right.$ fluence, around $20 \mathrm{~s}$, with the electron beam blocked) causes most particles to fragment extensively (Fig. 1c). A range of fragment sizes is visible, with the smallest about $2.5 \mathrm{~nm}$ in
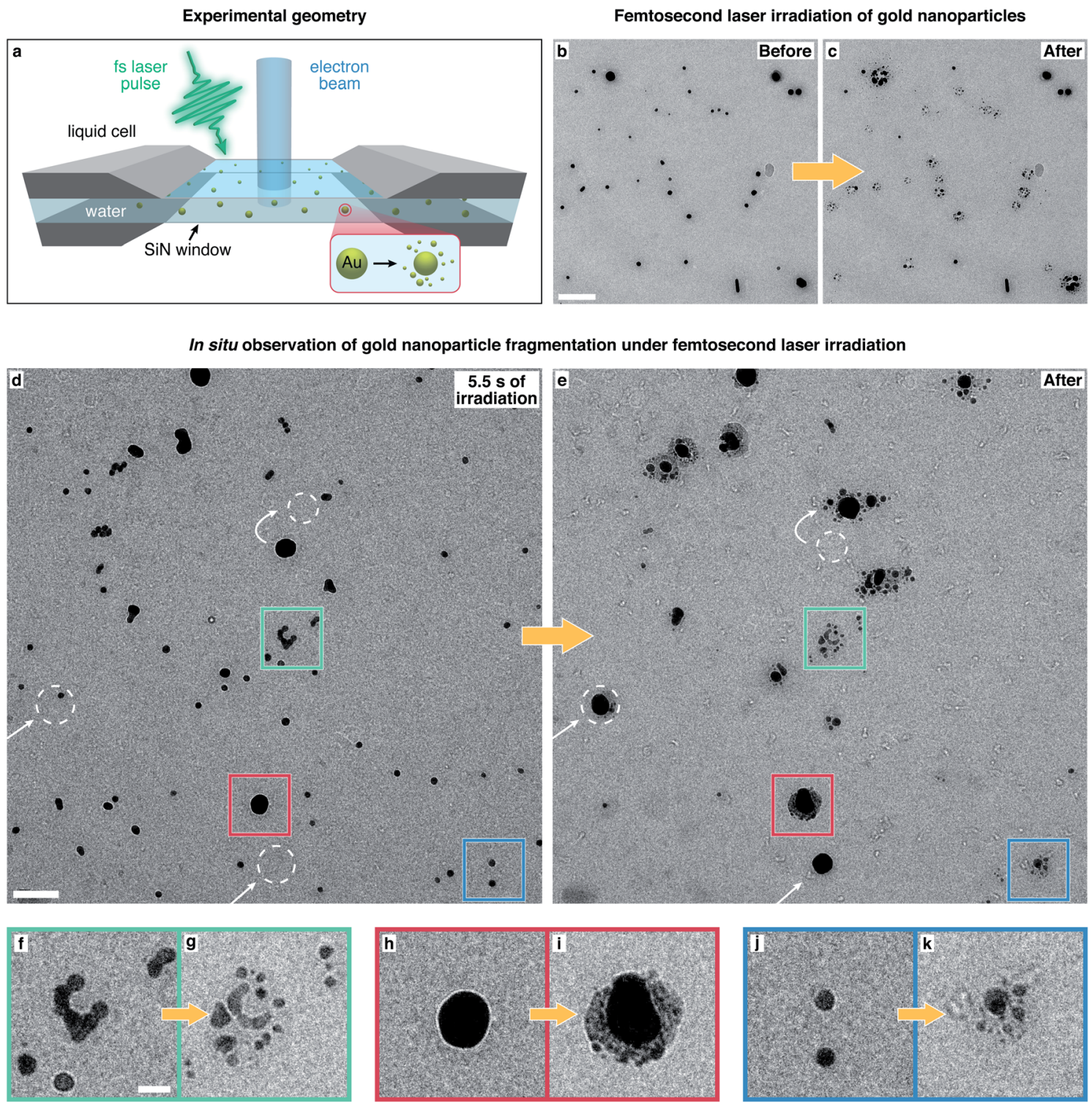

Fig. 1 In situ electron microscopy of the fragmentation of gold nanoparticles in water under femtosecond laser irradiation. (a) Sketch of the experimental geometry. Gold nanoparticles in water are enclosed in a liquid cell and are observed with the electron beam during irradiation with femtosecond laser pulses. ( $b$ and c) Micrographs of gold nanoparticles recorded before and after irradiation with femtosecond laser pulses (without simultaneous exposure to the electron beam), revealing that the laser induces heavy fragmentation. Scale bar, $200 \mathrm{~nm}$. (d and e) Images from a movie recorded during in situ laser irradiation (ESI Movie $1 \dagger$ ), showing the gold particles after $5.5 \mathrm{~s}$ of laser illumination (d) and after $30 \mathrm{~s}$ (e). White arrows and dashed circles indicate particles that moved into the field of view or diffused to a different position. scale bar, $100 \mathrm{~nm}$. ( $\mathrm{f}-\mathrm{k}$ ) Details of the areas marked with colored squares in (d) and (e). Scale bar, $25 \mathrm{~nm}$. 
diameter. In all our experiments, we have used the minimum fluence necessary to induce fragmentation. The fragmentation behavior is qualitatively the same for fluences up to $75 \mathrm{~mJ} \mathrm{~cm}{ }^{-2}$, at which point the liquid cell chip usually cracks.

Real-time observations of the fragmentation process allow us to shed light onto its mechanism, as shown in Fig. 1d-k (ESI Movie $1 \dagger$ ). We note that exposure to the electron beam at the low dose rates employed here (typically 3 electrons $\mathrm{A}^{-2} \mathrm{~s}^{-1}$ ) does not appear to alter the fragmentation pattern. Fig. 1d shows that after $5.5 \mathrm{~s}$ of laser irradiation $\left(53 \mathrm{~mJ} \mathrm{~cm}^{-2}\right)$, some particles have fused into larger structures, such as the one in the detail shown in Fig. 1f (green frame in Fig. 1d), indicating that at fluences high enough to induce fragmentation, the particles melt at least partially. Fragmentation usually sets in after several seconds of laser irradiation (7-10 s in ESI Movie $1 \dagger$ ), so that most particles in Fig. 1d are still intact. In contrast, extensive fragmentation is evident after $30 \mathrm{~s}$ of irradiation (Fig. 1e). Some particles (highlighted with white arrows and circles) have diffused to a different location or have appeared within the field of view, confirming that the particles are in a liquid film that enables their movement. In fact, fragmentation does not occur in the absence of water. This is illustrated in ESI Fig. 1, $\dagger$ which demonstrates that in partially dry samples, only particles located within patches of liquid undergo fragmentation.

ESI Movie $1 \dagger$ also reveals that Ostwald ripening ${ }^{39}$ occurs under laser irradiation, with the larger particles growing at the expense of the smaller ones, which shrink over time and have largely disappeared in Fig. 1e. This process, which appears to involve the solution-mediated transport of material, is induced by femtosecond laser irradiation. It occurs independently of whether the sample is simultaneously exposed to the electron beam, but cannot be induced through electron beam irradiation alone (see also ESI Fig. 2 and $3 \dagger$ ). Most of the remaining particles in Fig. 1e are surrounded by a large number of fragments. Details of the areas marked with colored squares are shown in Fig. 1f-k and highlight different fragmentation patterns. The creation of smaller fragments from larger particles is thermodynamically unfavorable and therefore points to a physical fragmentation mechanism that occurs simultaneously with the solution-mediated Ostwald ripening process.

Close inspection of the fragmentation process sheds further light onto the nature of the underlying mechanism. The image sequences in Fig. 2a-e depict the evolution of representative nanoparticles in Fig. 1e and d (ESI Movies 2-6†), with schematic illustrations of their transformations shown on the left. The timestamps refer to the time elapsed after laser irradiation started. Fig. 2a depicts a typical fragmentation process. Initially, two $15 \mathrm{~nm}$ diameter particles are visible (1) that fuse under laser irradiation (2) to form a spherical particle, which after $6.8 \mathrm{~s}$, ejects an individual progeny particle of $4.5 \mathrm{~nm}$ diameter (3). The parent particle then continues to shrink as additional progeny particles are ejected $(4,5)$. Fragmentation clearly does not involve an explosion, but rather a stepwise process. Individual progeny particles appear suddenly, from one movie frame to the next (200 ms frame duration), suggesting that they are ejected individually, in a process that closely resembles the femtosecond laser-induced fission of gold particles in a silica shell that we previously reported. ${ }^{30}$ After they first appear, some of the progeny particles slowly increase in size, suggesting that a solution-mediated growth process occurs. A frequently observed feature of the fragmentation process is that progeny particles surround the parent at a well-defined distance (Fig. 2a, frames 4,5$)$. We speculate that nanobubbles, which are expected to form under our experimental conditions, ${ }^{20-22}$ may play a role in creating this configuration.

While the defining feature of the fragmentation process is the stepwise ejection of progeny particles, complex fragmentation pathways can arise under prolonged laser irradiation. As the image sequence in Fig. $2 \mathrm{~b}$ illustrates, progeny particles frequently undergo fragmentation themselves. Two nanoparticles of 50 and $25 \mathrm{~nm}$ diameter (1) eject several fragments $(2,3)$, which then create second-generation progeny particles (4, $5)$. Fragmentation competes with the fusion of different particles (see ESI Movie $3 \dagger$ ), and an intricate fragmentation pattern is created, with particles of different sizes spread out in all directions. This is in marked contrast to the fission of gold particles in a silica shell, where prolonged irradiation leads to the formation of a binuclear particle. In a silica shell, progeny particles are preferentially ejected along the laser polarization direction and eventually coalesce into a second gold particle of equal size. It is connected to the first through a channel that forms in the silica shell that restricts the fission geometry. ${ }^{30}$ This is notably not the case in water, where fragments can spread in all directions.

The ejection of progeny particles occurs simultaneously with solution-mediated etching and growth processes. While lasermediated Ostwald ripening causes large particles such as those in Fig. $2 b$ to grow over time, smaller particles shrink as they undergo fragmentation and finally dissolve entirely. Fig. 2c shows an example of three $15 \mathrm{~nm}$ diameter particles, whose size initially remains constant until about $12 \mathrm{~s}$ of laser irradiation (1), when they begin to shrink. About 2-4 seconds later, each ejects several progeny particles that appear at similar distances from the parent (2-4). Under continued irradiation, parent and fragment particles finally dissolve completely (5). The appearance of fragments closely follows the moment at which the parent particles begin to shrink, suggesting that both phenomena are related or possibly even involve the same mechanism. We previously observed that gold particles encapsulated in a silica shell eject progeny droplets with as few as 10 atoms (0.7 $\mathrm{nm}$ diameter). ${ }^{30}$ Such small fragments would not offer sufficient contrast in the liquid cell environment. The ejection of clusters that are too small to be observable may therefore contribute to the etching of the particles in water. As shown in Fig. 2d, some particles develop a dark halo as they undergo fragmentation. This halo likely consists of a large number of small clusters and atoms that have been ejected from the parent.

Under prolonged irradiation, many particles enter a chaotic state of flux, in which repeated cycles of fragmentation and fusion occur, and etching and growth processes reshuffle mass between different particles and their fragments. Fig. 2e shows such an example. Three gold nanoparticles eject several fragments each $(1,2)$ and after several subsequent cycles of fission and fusion (3), merge into one large elongated particle (4), which then begins to eject progeny particles itself (5). 
Fragmentation pathways of gold nanoparticles
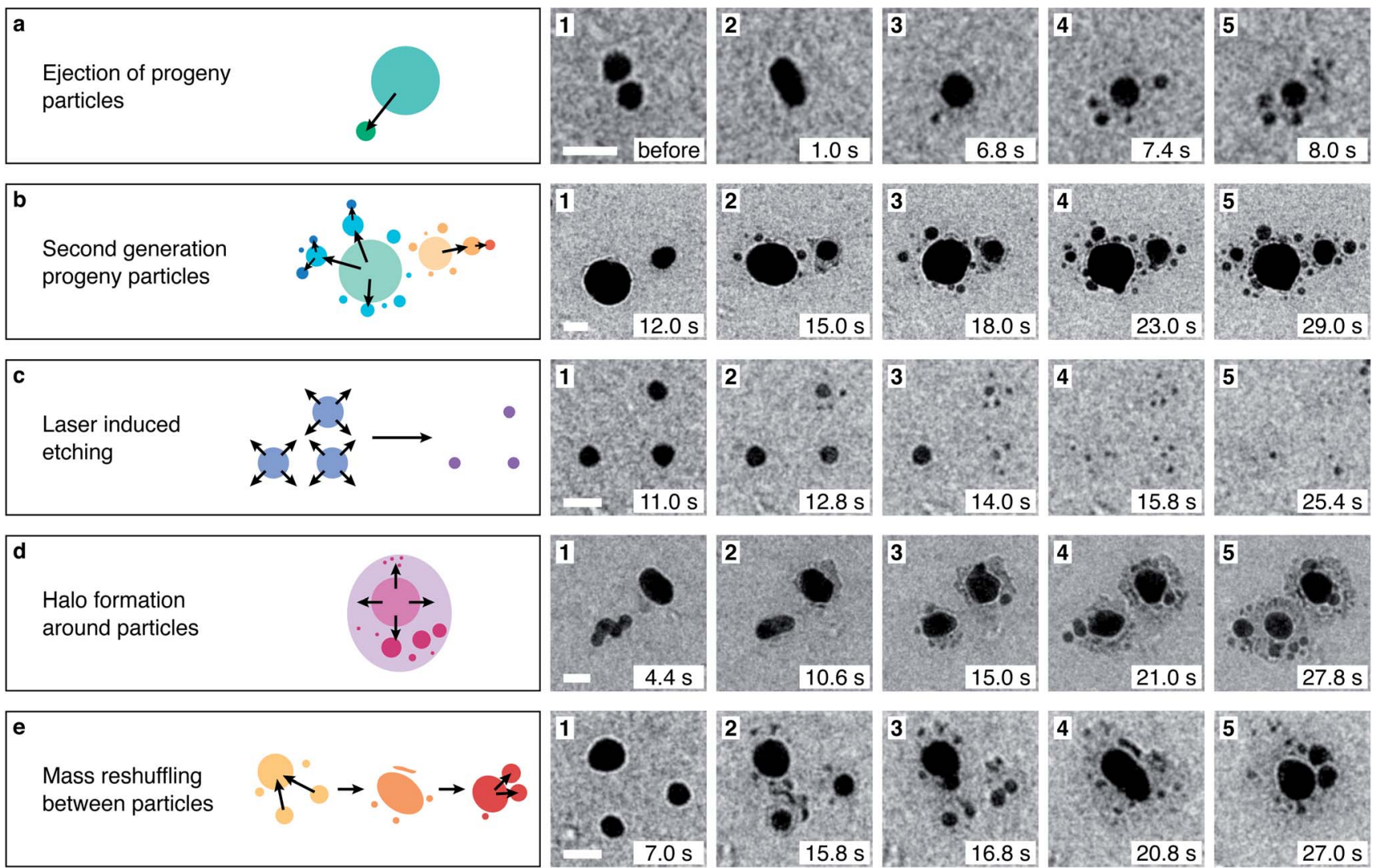

Fig. 2 Fragmentation pathways of gold nanoparticles in water under femtosecond laser irradiation. Image sequences illustrate representative transformations of the particles in the experiment of Fig. 1d and e (see ESI Movies 2-6†). A schematic representation of each process is provided on the left. (a) Two gold nanoparticles fuse into a particle of $13 \mathrm{~nm}$ diameter, which then ejects individual progenies of about 2-3 nm diameter in a stepwise process. (b) Progeny particles frequently undergo fragmentation themselves, a process that creates arrays of several generations of progenies. (c) Femtosecond laser irradiation dissolves smaller particles while growing the larger ones. Here, three small particles (10-12 nm diameter) fragment while slowly dissolving. (d) Some particles develop a dark halo over time that appears to consist of a large number of small ejected clusters. (e) Repeated cycles of fragmentation and fusion, as well as etching and growth reshuffle mass between different particles, creating rapidly fluctuating configurations. The time stamps of the micrographs refer to the time elapsed since the beginning of laser irradiation. scale bars, $30 \mathrm{~nm}$.

In contrast to the fission of gold nanoparticles encapsulated in a silica shell, ${ }^{30}$ the ejection of fragments from gold nanoparticles in water is not directional. Fig. 3a displays a micrograph of gold core silica shell nanoparticles $(20 \mathrm{~nm}$ core diameter and $20 \mathrm{~nm}$ shell thickness) after irradiation with femtosecond laser pulses $(100 \mathrm{~mJ}$ $\mathrm{cm}^{-2}$, about $30 \mathrm{~s}$ ). Cavities in the silica shell have formed around the gold cores, and several fragments (1-2 nm diameter) have been ejected. Fig. $3 c$ shows a histogram of the ejection angle $\theta$, as defined in Fig. 3a. The ejection of progeny particles is highly directional and as previously reported, preferentially occurs in the laser polarization direction (horizontal). ${ }^{30} \mathrm{~A} \cos ^{4}(\theta)$ fit of the angular distribution (red curve) therefore allows us to accurately determine the laser polarization direction, ${ }^{30,40}$ which is indicated with a double-headed arrow in Fig. 3a. In contrast, gold nanoparticles in water (Fig. 3b) eject fragments under femtosecond laser irradiation $\left(53 \mathrm{~mJ} \mathrm{~cm} \mathrm{~cm}^{-2}\right)$ without any apparent angular preference (Fig. 3d).

Our observations clearly contradict a fragmentation mechanism involving either a Coulomb or thermal explosion. They are also not consistent with processes that typically occur at much higher fluence and violently shatter the particle, such as nearfield ablation or shockwave-mediated disintegration. Instead, progeny particles are emitted one after another, and ejection is a rare event that on average occurs once every several thousand laser pulses. This stepwise fragmentation process closely resembles that of gold nanoparticles in a silica shell, suggesting that it involves the same mechanism, Coulomb fission. In both cases, the fragmentation of $20 \mathrm{~nm}$ gold particles requires an almost identical absorbed fluence that causes the particles to melt, and progeny particles of similar size are generated (as discussed above, particles smaller than $2 \mathrm{~nm}$ are not observable in water). Most tellingly, fragmentation appears to involve the ionization of the parent particles. This is demonstrated in ESI Fig. 2 and 3 (ESI Movies 7-9 and 10-14†), which show that nanoparticles that eject progenies under femtosecond irradiation do not fragment any further under subsequent exposure to nanosecond pulses of up to 4.5 times the fluence $(532 \mathrm{~nm}, 0.7$ ns pulse duration, $10 \mathrm{kHz}$ ). The particles continue to undergo shape changes, as they do under femtosecond irradiation, 


\section{Angular distribution of progeny particles}

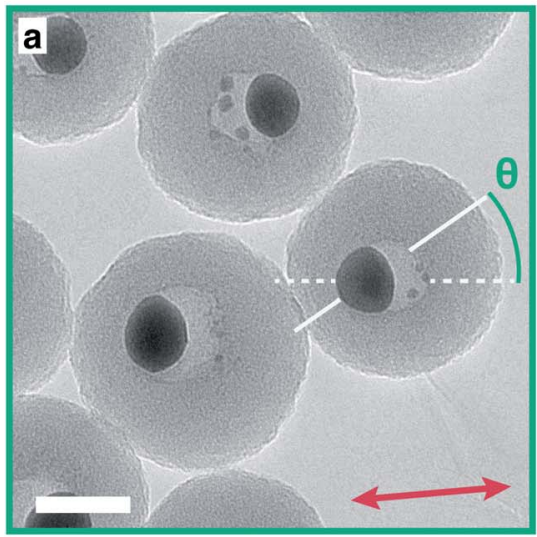

Gold particles in silica shell
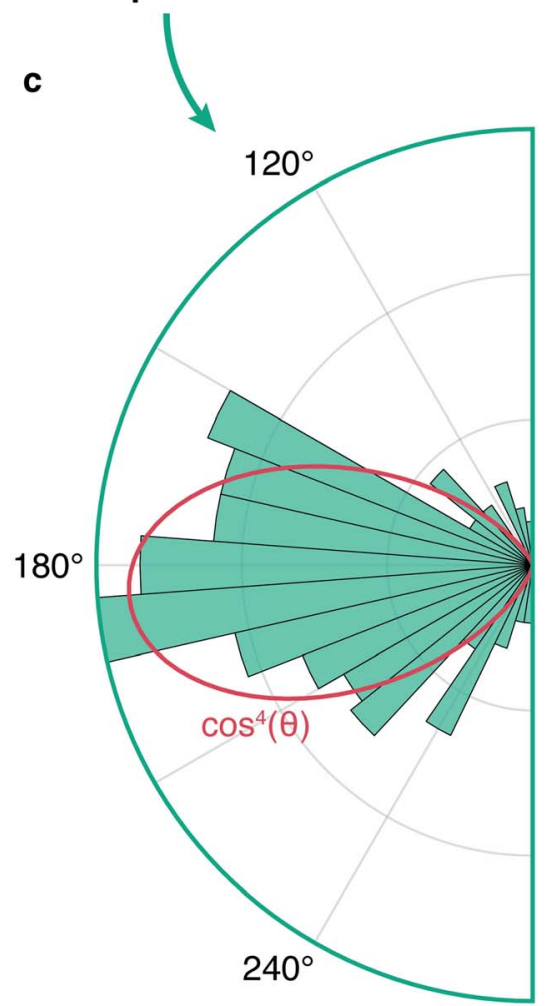

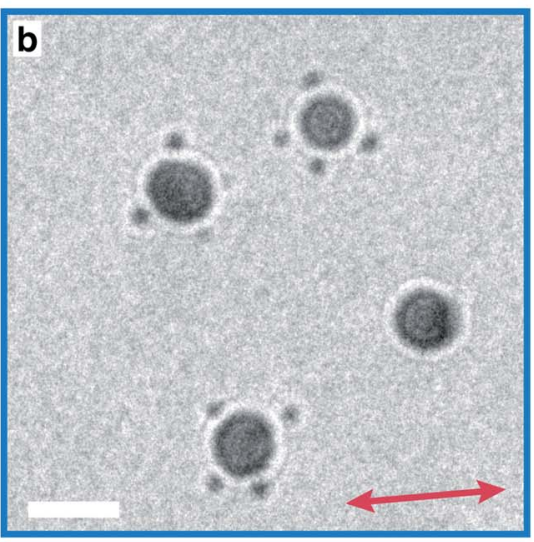

Gold particles in water

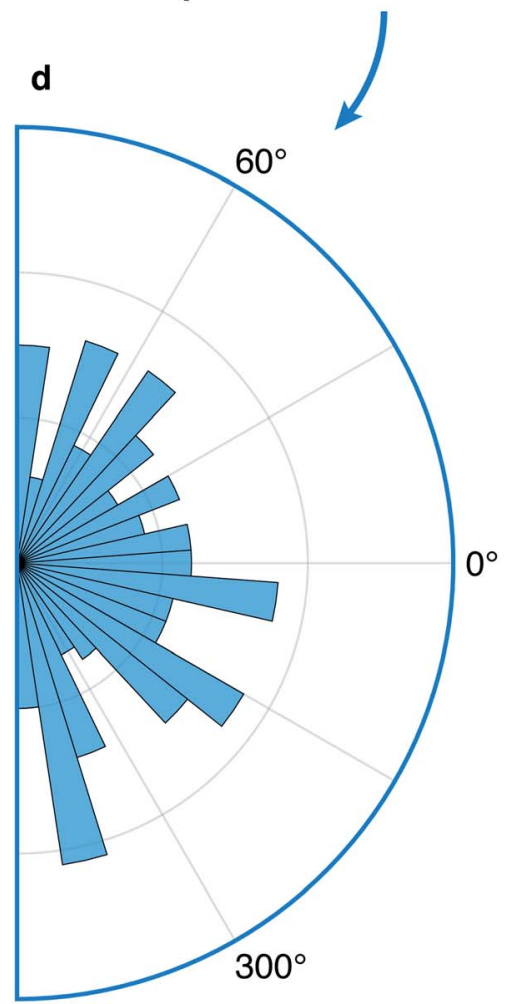

Fig. 3 Determination of the angular distribution of the progeny particles. ( $a$ and b) Micrographs of gold particles and their progenies ejected under femtosecond laser irradiation, for gold particles enclosed in a silica shell (a) and for gold particles in water (b). The definition of the ejection angle $\theta$ is indicated in (a) together with the laser polarization direction (double-headed arrow). (c and d) Angular distributions of the progeny particles. For gold particles in a silica shell, the distribution strongly peaks along the laser polarization direction (c), while it is isotropic for particles in water (d). $A \cos ^{4}(\theta)$ fit of the angular distribution (red curve) in (c) is used to determine the laser polarization direction indicated in (a). Scale bars, $25 \mathrm{~nm}$.

suggesting that they become at least partially liquid and reach similar temperatures. At the highest fluence (ESI Movie 9†), they melt into spheres whose outline fluctuates under continued irradiation. Yet, no fragmentation occurs. This strongly suggests that the fragmentation mechanism involves the multiphoton ionization of the gold particles, which is known to occur at the femtosecond laser fluences used here, ${ }^{35,36,41}$ whereas nanosecond laser pulses only heat the particles without ionizing them. ${ }^{25}$ Evidently, the ejection of individual progeny particles occurs from an ionized, liquid parent particle, which strongly suggests that Coulomb fission is the primary fragmentation mechanism, a conclusion that is consistent with an analysis of the energetics of different fragmentation pathways. ${ }^{6}$

Whereas gold particles in a silica shell preferentially emit progeny particles in the laser polarization direction, no angular preference exists in water. We propose that this is due to the different electrical conductivities of the surrounding media. During femtosecond laser ionization of plasmonic 

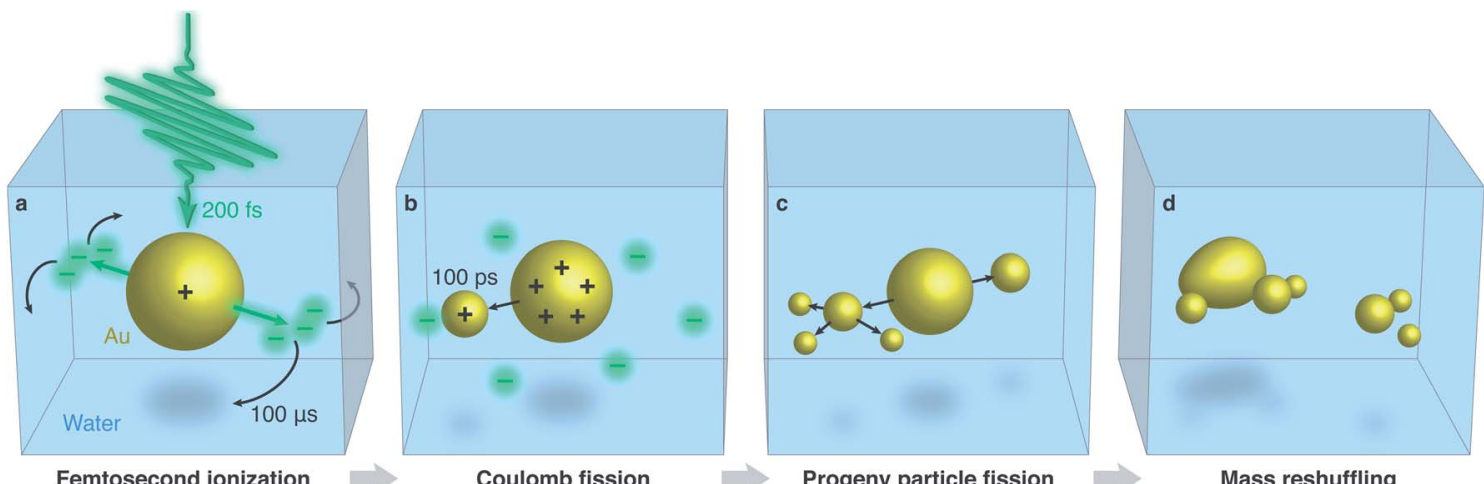

Fig. 4 Proposed mechanism of the femtosecond laser-induced fragmentation of gold nanoparticles in water. (a) Under irradiation with a femtosecond laser pulse, the gold particle is ionized and electrons are emitted in the laser polarization direction. Diffusion of the solvated electrons rapidly establishes an isotropic charge distribution. (b) Under continued irradiation, the melted gold particle undergoes Coulomb fission, ejecting a highly charged progeny particle in a random direction. (c) Further fission events occur, and second-generation progeny particles are formed. (d) The particles enter a state of flux as fragments undergo repeated cycles of fission and fusion, while solution-mediated etching and growth processes reshuffle mass between different particles.

nanoparticles, photoelectron emission predominantly occurs in the laser polarization direction. ${ }^{35,36,41}$ This creates an anisotropic distribution of negative charge in the silica shell that has a lifetime of seconds ${ }^{42,43}$ and is thus able to steer the emission of the positively charged progeny droplets. In contrast, electrons have a much higher mobility in water. Since the diffusion length of the solvated electron ${ }^{44}$ is larger than $1 \mu \mathrm{m}$ in $100 \mu \mathrm{s}$, the time between two laser pulses, an isotropic charge distribution is established before the next laser pulse arrives. Once the particle reaches the Rayleigh limit under continued laser irradiation, Coulomb fission occurs, and a progeny particle is ejected in a random direction.

The physical fragmentation of the gold nanoparticles is accompanied by solution-mediated etching and growth. These processes, which also allow Ostwald ripening to take place, are reminiscent of the plasmon-mediated chemistry that occurs under irradiation with continuous lasers. ${ }^{11-14}$ Hot electrons that are created through plasmon excitation reduce gold ions present in solution, allowing the nanoparticle to grow, while hot holes cause gold ions to dissolve, providing a competing pathway for particles to be etched. It is conceivable that a similar mechanism operates in our experiments. However, no significant etching or growth takes place under irradiation with nanosecond pulses, even of several times higher fluence (ESI Fig. 2 and 3 as well as ESI Movies 7-9 and 10-14†). This suggests that the dissolution of particles in our experiments entails their ionization. It also raises the possibility that etching is actually not a distinct phenomenon, but rather involves the ejection of clusters that are too small to be observed and that occurs as the particle undergoes Coulomb fission.

Fig. 4 summarizes the proposed fragmentation mechanism. Irradiation with a femtosecond laser pulse (200 fs) ionizes the gold particle, with photoelectrons preferentially emitted in the laser polarization direction (Fig. 4a). Rapid diffusion of the solvated electrons then leads to an isotropic charge distribution before the next laser pulse arrives $(100 \mu \mathrm{s}$ later). Under continued irradiation, the gold particle is charged to the Rayleigh limit. As it is melted by a laser pulse, it undergoes
Coulomb fission by ejecting a highly charged progeny particle in a random direction (Fig. $4 \mathrm{~b}$ ), a process that occurs on a timescale of about 100 ps (ref. 45). A complex fragmentation pattern arises as further progeny particles are ejected and undergo fission themselves (Fig. 4c). Under prolonged irradiation, a state of flux results as the fragments undergo repeated cycles of fission and fusion that are accompanied by solution-mediated etching and growth processes.

\section{Conclusions}

In conclusion, our observations provide evidence that Coulomb fission is the primary mechanism of the femtosecond laserinduced fragmentation of gold nanoparticles in water. Using in situ electron microscopy, we reveal that prolonged irradiation gives rise to complex, multistep fragmentation pathways that are accompanied by solution-mediated etching and growth processes. Our experiments highlight the importance of in situ observations for understanding and disentangling the multitude of interactions of plasmonic particles with laser radiation.

\section{Data availability}

The data that support the findings of this study are available from the corresponding author upon request.

\section{Author contributions}

U. J. L. conceived the experiments. M. D. and U. J. L. supervised the project. G. B., P. K. O., and C. Y. prepared samples and performed the experiments. G. B., P. K. O., C. Y., J. M. V., and U. J. L. analyzed the data. All authors contributed to the preparation of the manuscript.

\section{Conflicts of interest}

The authors declare no competing financial interests. 


\section{Acknowledgements}

This work was supported by the ERC Starting Grant 759145 and by the Swiss National Science Foundation Grants PP00P2_163681 and 206021_183295.

\section{References}

1 D. Werner, A. Furube, T. Okamoto and S. Hashimoto, J. Phys. Chem. C, 2011, 115, 8503-8512.

2 S. Besner, A. V. Kabashin and M. Meunier, Appl. Phys. Lett., 2006, 89, 233122.

3 K. Maximova, A. Aristov, M. Sentis and A. V. Kabashin, Nanotechnology, 2015, 26, 065601.

4 F. A. Videla, G. A. Torchia, D. C. Schinca, L. B. Scaffardi, P. Moreno, C. Méndez, L. J. Giovanetti, J. M. Ramallo Lopez and L. Roso, J. Appl. Phys., 2010, 107, 114308.

5 O. Warshavski, L. Minai, G. Bisker and D. Yelin, J. Phys. Chem. C, 2011, 115, 3910-3917.

6 L. Delfour and T. E. Itina, J. Phys. Chem. C, 2015, 119, 1389313900.

7 J. M. Dziedzic, J. E. Bjorkholm and S. Chu, Opt. Lett., 1986, 11, 288-290.

8 D. G. Grier, Nature, 2003, 424, 810-816.

9 S. Wang and T. Ding, ACS Nano, 2019, 13, 32-37.

10 W. Xie and S. Schlücker, Nat. Commun., 2015, 6, 7570.

11 Y. Zhang, S. He, W. Guo, Y. Hu, J. Huang, J. R. Mulcahy and W. D. Wei, Chem. Rev., 2018, 118, 2927-2954.

12 Y. Long, S. Wang, Y. Wang, F. Deng and T. Ding, J. Phys. Chem. C, 2020, 124, 19212-19218.

13 M. R. Langille, M. L. Personick and C. A. Mirkin, Angew. Chem., Int. Ed., 2013, 52, 13910-13940.

14 Y. Zhai, J. S. DuChene, Y.-C. Wang, J. Qiu, A. C. JohnstonPeck, B. You, W. Guo, B. DiCiaccio, K. Qian, E. W. Zhao, F. Ooi, D. Hu, D. Su, E. A. Stach, Z. Zhu and W. D. Wei, Nat. Mater., 2016, 15, 889-895.

15 G. González-Rubio, P. Díaz-Núñez, A. Rivera, A. Prada, G. Tardajos, J. González-Izquierdo, L. Bañares, P. Llombart, L. G. Macdowell, M. Alcolea Palafox, L. M. LizMarzán, O. Peña-Rodríguez and A. Guerrero-Martínez, Science, 2017, 358, 640-644.

16 G. González-Rubio, J. González-Izquierdo, L. Bañares, G. Tardajos, A. Rivera, T. Altantzis, S. Bals, O. PeñaRodríguez, A. Guerrero-Martínez and L. M. Liz-Marzán, Nano Lett., 2015, 15, 8282-8288.

17 T. Milagres de Oliveira, W. Albrecht, G. González-Rubio, T. Altantzis, I. P. Lobato Hoyos, A. Béché, S. Van Aert, A. Guerrero-Martínez, L. M. Liz-Marzán and S. Bals, ACS Nano, 2020, 14, 12558-12570.

18 G. González-Rubio, A. Guerrero-Martínez and L. M. LizMarzán, Acc. Chem. Res., 2016, 49, 678-686.

19 S. Hashimoto, D. Werner and T. Uwada, J. Photochem. Photobiol., C, 2012, 13, 28-54.

20 A. R. Ziefuss, S. Reich, S. Reichenberger, M. Levantino and A. Plech, Phys. Chem. Chem. Phys., 2020, 22, 4993.

21 T. Katayama, K. Setoura, D. Werner, H. Miyasaka and S. Hashimoto, Langmuir, 2014, 30, 9504-9513.
22 S. Hashimoto, T. Katayama, K. Setoura, M. Strasser, T. Uwada and H. Miyasaka, Phys. Chem. Chem. Phys., 2016, 18, 4994-5004.

23 S. Besner, A. V. Kabashin and M. Meunier, Appl. Phys. A, 2007, 88, 269-272.

24 S. Inasawa, M. Sugiyama and Y. Yamaguchi, J. Phys. Chem. B, 2005, 109, 9404-9410.

25 S. Hashimoto, D. Werner and T. Uwada, J. Photochem. Photobiol., C, 2012, 13, 28-54.

26 A. Pyatenko, M. Yamaguchi and M. Suzuki, J. Phys. Chem. C, 2009, 113, 9078-9085.

27 A. Takami, H. Kurita and S. Koda, J. Phys. Chem. B, 1999, 103, 1226-1232.

28 A. Plech, V. Kotaidis, M. Lorenc and J. Boneberg, Nat. Phys., 2006, 2, 44-47.

29 Y. Ihm, D. H. Cho, D. Sung, D. Nam, C. Jung, T. Sato, S. Kim, J. Park, S. Kim, M. Gallagher-Jones, Y. Kim, R. Xu, S. Owada, J. H. Shim, K. Tono, M. Yabashi, T. Ishikawa, J. Miao, D. Y. Noh and C. Song, Nat. Commun., 2019, 10, 2411.

30 J. M. Voss, P. K. Olshin, R. Charbonnier, M. Drabbels and U. J. Lorenz, ACS Nano, 2019, 13, 12445-12451.

31 F. Banhart, in In-Situ Electron Microscopy, ed. G. Dehm, J. M. Howe and J. Zweck, Wiley-VCH Verlag GmbH \& Co. KGaA, Weinheim, Germany, 2012, pp. 123-143.

32 D. Duft, T. Achtzehn, R. Müller, B. A. Huber and T. Leisner, Nature, 2003, 421, 128.

33 J. C. Burton and P. Taborek, Phys. Rev. Lett., 2011, 106, 144501.

34 S. Consta, M. Sharawy, M. I. Oh and A. Malevanets, Anal. Chem., 2017, 89, 8192-8202.

35 R. G. Hobbs, W. P. Putnam, A. Fallahi, Y. Yang, F. X. Kärtner and K. K. Berggren, Nano Lett., 2017, 17, 6069-6076.

36 W. P. Putnam, R. G. Hobbs, P. D. Keathley, K. K. Berggren and F. X. Kärtner, Nat. Phys., 2017, 13, 335-339.

37 L. Rayleigh, London, Edinburgh Dublin Philos. Mag. J. Sci., 1882, 14, 184-186.

38 E. A. Ring and N. de Jonge, Microsc. Microanal., 2010, 16, 622-629.

39 T. Tadros, in Encyclopedia of Colloid and Interface Science, Springer, Berlin, 2013, p. 820.

40 M. Sivis, N. Pazos-Perez, R. Yu, R. Alvarez-Puebla, F. J. García de Abajo and C. Ropers, Commun. Phys., 2018, 1, 13.

41 R. G. Hobbs, Y. Yang, A. Fallahi, P. D. Keathley, E. De Leo, F. X. Kärtner, W. S. Graves and K. K. Berggren, ACS Nano, 2014, 8, 11474-11482.

42 J. G. Mihaychuk, J. Bloch, Y. Liu and H. M. van Driel, Opt. Lett., 1995, 20, 2063.

43 J. G. Mihaychuk, N. Shamir and H. M. van Driel, Phys. Rev. B: Condens. Matter Mater. Phys., 1999, 59, 2164-2173.

44 G. V. Buxton, C. L. Greenstock, W. P. Helman and A. B. Ross, J. Phys. Chem. Ref. Data, 1988, 17, 513-886.

45 J. C. Burton and P. Taborek, Phys. Rev. Lett., 2011, 106, 144501.

46 P. K. Olshin, M. Drabbels and U. J. Lorenz, Struct. Dyn., 2020, 7, 054304. 